\title{
THE TEMPERATURE OF THE DIMETHYLHYDRAZINE DROPS MOVING IN THE ATMOSPHERE AFTER DEPRESSURIZATION OF THE FUEL TANK ROCKETS
}

\author{
Elena Bulba ${ }^{1, *}$, Tatiana Nemova $^{2}$, and Anna Dyatchina ${ }^{1}$ \\ ${ }^{1}$ National Research Tomsk Polytechnic University, 634050 Tomsk, Russia \\ ${ }^{2}$ Tomsk State Architecture and Civil Engineering, 634050 Tomsk, Russia
}

\begin{abstract}
This work includes the results of the numerical modeling of temperature changes process of the dimethylhydrazine (DMH) drops, taking into account the radial temperature gradient in the air after the depressurization of the fuel compartments rockets at high altitude. There is formulated a mathematical model describing the process of DMH drops thermal state modifying when it's moving to the Earth's surface. There is the evaluation of the influence of the characteristic size of heptyl drops on the temperature distribution. It's established that the temperatures of the small size droplets practically completely coincide with the distribution of temperature in the atmosphere at altitudes of up to 40 kilometers.
\end{abstract}

\section{Introduction}

The Liquid propellant rocket engines, most likely, will be the main engines for the heavy launch vehicles in the next 10-15 years. The main negative factor in the operation of such engines is their fuel - unsymmetrical dimethylhydrazine (heptyl) which may fall on the Earth's surface after the depressurization of the engine exhaust fuel tanks [1]. Until recently, there is not a valid opinion on the possibility of heptyl contamination of soil and water of the territories over which the exhaust engine is separated from the spacecraft, launched into outer space. There are attempts to modeling processes of atmospheric diffusion of heptyl vapors and droplets in the surface layers of the atmosphere. [2,3] But the problem of evaporation of heptyl in the atmosphere while driving to the ground in a production that takes into account all the major significant factors not solved so far. One of such factors is the thermal state of this liquid fuel droplets in the air (the surface temperature determines the rate of evaporation of heptyl [3]). It is known the solvings of the problems of the temperature field of this fuel droplets by assuming its homogeneity [2] and in the description of the temperature distribution in a drop using the heat equation in partial derivatives [3]. But these studies [3] were carried excluding the complex [4]

${ }^{*}$ Corresponding author: bulba@tpu.ru 
distribution of temperature over the height of the atmospheric layer, which must have an important role in the formation of a drop temperature field and, consequently, the evaporation rate of heptyl.

\section{Statement of the problem}

The mathematical formulation of the problem of the formation of a small size spherical form drop temperature field is reduced to the solution of the heat equation for a sphere in the form of

$$
\rho c \frac{\partial T}{\partial t}=\frac{1}{r^{2}} \frac{\partial}{\partial r}\left(\lambda r^{2} \frac{\partial T}{\partial r}\right)
$$

with the initial condition

$$
t=0, \quad T=T_{0}
$$

and with the boundary conditions

$$
\begin{aligned}
r=R_{\mathrm{\kappa}}:-\lambda \frac{\partial T}{\partial r} & =\alpha\left(T^{e}-T\right), t>0, \alpha>0, \\
r & =0 \quad \frac{\partial T}{\partial r}=0,
\end{aligned}
$$

Where $\rho$ - density of droplets, $\mathrm{c}$ - the specific heat capacity, $\lambda$ - coefficient of thermal conductivity, $\mathrm{r}$ - radial coordinate, $R_{\mathrm{K}}$ - outer radius of the droplets, $\mathrm{T}$ - temperature, $T_{0}$ initial value of the drop temperature, $T^{e}$ - ambient temperature, $\alpha$ - heat transfer coefficient.

The communication between ambient temperature and height [4] is presented in Table 1.

Table 1. Changing atmospheric temperature with height.

\begin{tabular}{|c|c|c|c|c|c|c|c|c|}
\hline $\begin{array}{c}\text { Outside } \\
\text { Temperature, } \mathrm{K}\end{array}$ & 220 & 220 & 233 & 250 & 273 & 255 & 233 & 206 \\
\hline Height, $\mathrm{m}$ & 10000 & 20000 & 30000 & 40000 & 50000 & 60000 & 70000 & 80000 \\
\hline
\end{tabular}

The calculation of the drop temperature is necessary to determine the speed of the fall of $v$ in the equation of its motion, taking into account the effect of air resistance. It is also necessary to know the velocity $v$ to calculate the heat transfer coefficient $\alpha$. The equation of motion of the spherical form drop is as follows:

with the initial condition

$$
\frac{d v}{d t}=g-\frac{3 C_{D} \rho_{\mathrm{B}}}{4 d \rho_{\mathrm{r}}}\left(v-v_{\mathrm{B}}\right)^{2}
$$

$$
t=0, \quad v=0,
$$

where $v$ - velocity of the fall of drops; $v_{\mathrm{B}}$ - air velocity; $\rho_{\mathrm{B}}$ - density of the air pollution; $\rho_{\mathrm{r}}$ the density of the liquid heptyl; $d$ - droplet diameter; $C_{D}$ - coefficient of resistance; $g$ acceleration of gravity.

The rate of air flow is assumed to be 0 .

The drag coefficient of the particle $C_{D}$ in the turbulent regime accepted constant value of 0.44. In general, any liquid droplet in flight by gravity force is deforming. In a process of deformation the various forms of a drop are possible (sphere, ellipsoid, "pancake", "pear" et al. $[6,7])$. In experiments $[6,7]$ was found that even during the time of relatively small 
flight distances of 1-2 meters the water, kerosene and acetone droplet goes through several cycles of such deformations changing its form. But with the growth speed of flight any liquid droplets are increasingly taking the form of a sphere. At the same time, less is the characteristic size of the drops, so the greater flight time it retains the shape of a sphere. That's why the spherical shape of the drop and the corresponding coefficient of resistance are adopted for the mathematical modeling. The heat transfer coefficient from the particles obtained values fall rate was calculated from the expressions

$$
\begin{gathered}
\mathrm{Nu}=2+0,27 \operatorname{Re}^{0.62} \operatorname{Pr}^{0.33}, \\
\alpha=\frac{\lambda \mathrm{Nu}}{d} .
\end{gathered}
$$

There Nu, Re, Pr - are Nusselt number, Reynolds number, Prandtl number, respectively. Prandtl number was assumed to be 0.7 , and the Reynolds number was calculated using the equation obtained from (4) the values of the velocities for the respective points in time. The kinematic viscosity coefficient is adopted in accordance with the tabulated data [4].

\section{Results and discussion}

The results of numerical solution of the problem (1) - (8) are shown in figure 1. There are presented the dependences of a heptyl drops temperature on a height of three specific sizes. It also includes a comparison with the results obtained previously in [2], excluding the radius droplet temperature gradient.

The numerical analysis shows that at small droplet sizes characteristic (curves 4, 6) there is a coincidence of a heptyl and air temperature over the entire height of incidence (curve 3). For large drops (curve 1), these temperatures are significantly different. Therefore, we can conclude that the temperature of characteristic small size droplets can be considered identical to the air temperature at the respective altitude. But for the larger heptyl drops, with greater thermal inertia and a high-speed fall, it is necessary to take into account the distribution of temperature drop by drop.

The comparisons with the previous [2] results (figure 1) shows that at small characteristic size of drops (curves 4,6 , and curves 5, 7, curve 3), their temperatures are almost identical. For the large heptyl droplets model (1) - (8) clarifies established in [2] the temperature distribution.

The theoretical investigation illustrates the thermal state of the heptyl drops driving in the atmosphere. After the depressurization of the fuel tanks of launch vehicles spacecraft airborne cloud descending to the surface of the Earth is changing. The larger drops, flying some distance, are spliting into smaller ones. The calculation results show that the larger is the droplet size, then the higher is the temperature and a possibly rate of evaporation at high altitudes. Small droplets also fall at a slower rate. Heptyl cloud, which may be imposed on long distance, is forming at high altitudes.

To derive the possibility of heptyl drops precipitation on the surface of the Earth is necessary to estimate the rate of evaporation of the fuel at the set value of the drops temperature at different heights. This can be done using the device [8.9]. But for modeling heptyl evaporation process it requires the data on the coefficients of evaporation [8] of the liquid fuel, which has not yet been established. Therefore, one of the tasks, the solution of which is necessary to conclude about the possibility of loss of heptyl in the Earth's surface, is to define the phase transformation characteristics of this fuel in the temperature range corresponding to the Earth's atmosphere. 


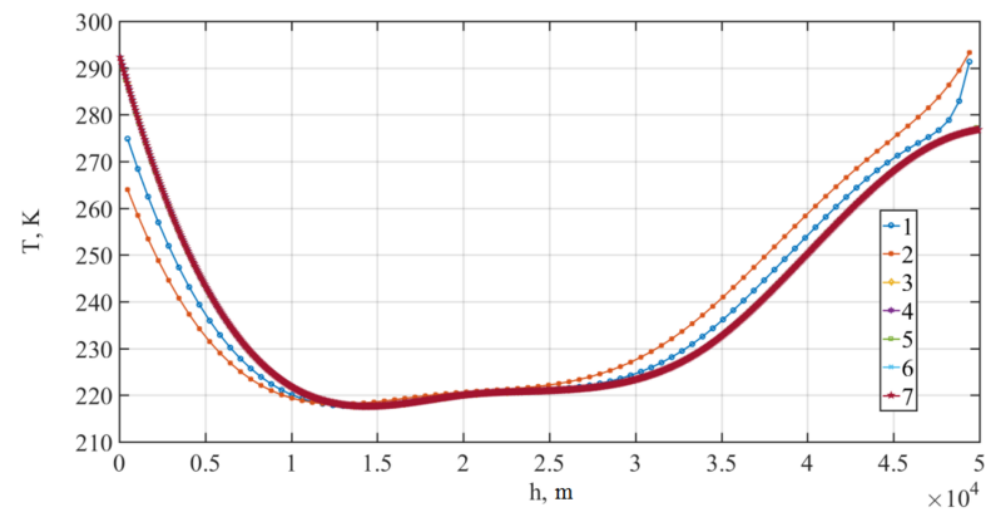

Fig. 1. The temperature of a heptyl spherical droplet with a characteristic size: $1-\mathrm{d}=10 \mathrm{~mm}$, taking into account the temperature gradient along the radius; $2-\mathrm{d}=10 \mathrm{~mm}$ without radial temperature gradient; 3 - the temperature drop over the entire height; $4-\mathrm{d}=1 \mathrm{~mm}$, taking into account the radial temperature gradient; $5-\mathrm{d}=1 \mathrm{~mm}$ without the temperature gradient along the radius; $6-\mathrm{d}=1 \mathrm{~mm}$, taking into account the temperature gradient along the radius; $7-\mathrm{d}=1 \mathrm{~mm}$ without the temperature gradient along the radius.

\section{Conclusion}

The resulting numerical studies of the temperature distribution in height of falling drops of DMH based on the radius of the spherical particles of the temperature gradient for the large drops illustrate the effect of the characteristic size on the thermal processes occurring in the droplets. It is found that the temperature of the small size droplets almost completely coincide with the air temperatures over the entire height of heptyl incidence.

\section{Acknowledgments}

The work was supported by the Russian Federation President's grant (NSH project 7538.2016.8).

\section{References}

1. E.L. Aleksandrov, Ecological problems of the effects and risks of space engineering on the environment: A Reference Guide (ANKIL, Moscow, 2000)

2. G.V. Kuznetsov, T.N. Nemov, E.E. Bulba, Izv.vuzov. Physics 6 (2006)

3. A.E. Dolotov, G.V. Kuznetsov, T.N. Nemova, Izv.vuzov. Physics 4 (2008)

4. Standard aktmosfera. Options. - M .: State Committee of the Council of Ministers of the USSR standards (1977)

5. V.I. Terehov, M.A. Pahomov, Heat and mass transfer and hydrodynamics in a gasdrop flow (Monograph NGTU. Novosiboirsk, 2008)

6. R.S. Volkov, G.V. Kuznetsov, P.A. Strizhak, Int. J. Heat Mass Transfer 85 (2015)

7. R.S. Volkov, O.V. Vysokomornaya, G.V. Kuznetsov, P.A. Strizhak, Tech. Phys. Lett. 41, 2 (2015)

8. G.V. Kuznetsov, P.A. Strizhak, J. Eng. Thermophys. 17, 3 (2008)

9. R.S. Volkov, G.V. Kuznetsov, P.A. Strizhak, Tech. Phys. Lett. 59, 7 (2014) 